\title{
Surface electronic structure and magnetic properties of doped manganites
}

\author{
M. J. Calderón ${ }^{1,2}$, L. Brey ${ }^{1}$ and F. Guinea ${ }^{1}$ \\ ${ }^{1}$ Instituto de Ciencia de Materiales (CSIC). Cantoblanco, 28049 Madrid. Spain. \\ ${ }^{2}$ Departamento de Fúsica Teórica de la Materia Condensada, Universidad Autónoma de Madrid, 28049 Madrid. Spain.
}

(June 4, 2018)

\begin{abstract}
The electronic structure and magnetic properties of $\mathrm{La}_{1-x} \mathrm{~A}_{x} \mathrm{MnO}_{3}$ are investigated. It is assumed that, at the outermost layer, the environment of the Mn ions does not have cubic symmetry. The $e_{g}$ orbitals are split and the double exchange mechanism is weakened. The charge state of the Mn ions is modified, and the magnetic ordering of the spins tends to be antiferromagnetic. The surface magnetization and the dependence of the transport properties through the resulting surface barrier on applied magnetic field and temperature is analyzed.
\end{abstract}

75.20.Ss,71.20.Lp,75.30.Et,75.30.Pd

Doped manganites show many unusual features, the most striking being the colossal magnetoresistance in the fully ferromagnetic phase [1]2]. Extensive research shows that the transport properties, and the magnetoresistance in particular, are significantly modified at artificially created barriers [3 10] or in ceramic materials $11-15$. The magnetoresistance, as function of temperature, drops more rapidly than in the bulk. It has larger values at low fields, and persists at large fields, unlike in the bulk case. The relevance of the interfaces in perovskite manganites can also be inferred by comparing with transport in related materials which exhibit colossal magnetoresistance 116]. These properties have been ascribed to changes in the interface structure, although the origin of these modifications, and the resulting structure are not known.

In the present paper, we analyze the simplest, and most common, modification with respect to the bulk that a surface may show: the loss of cubic symmetry around the $\mathrm{Mn}$ ions. It is well known that $\mathrm{La}_{1-x} \mathrm{~A}_{x} \mathrm{MnO}_{3}$ shows a transition from a tetragonal (or orthorhombic) structure to a cubic one as the value of the doping $x$ is increased. The systems with the highest Curie temperature have $x \sim \frac{1}{3}$ and are in the cubic phase. This implies that the two $e_{g}$ orbitals of the Mn ion are degenerate and contribute to the conduction band. In this situation, the double exchange mechanism is enhanced.

The cubic symmetry is lost at the surface. If the last layer is oxygen deficient, the resulting splitting between the $e_{g}$ orbitals can be larger than typical Jahn-Teller splittings in $\mathrm{La}_{1-x} \mathrm{~A}_{x} \mathrm{MnO}_{3}$ with small values of $x$. When one of the $e_{g}$ orbitals moves away from the Fermi level, the double exchange mechanism is weakened, and direct antiferromagnetic couplings between the core $S=\frac{3}{2}$ spins can prevail. Moreover, the reduction in electronic kinetic energy can also lead to charge transfer between the surface layers and the bulk, contributing to the formation of a surface dipole. All these effects can be modified by surface spin waves, which, in turn, depend on temperature and external magnetic fields. A significant dependence of the metal-insulator transition temperature as function of oxygen pressure in thin films is found in [17].

In order to investigate these features, we start from a tight binding Hamiltonian, using the two $e_{g}$ orbitals, $d_{x^{2}-y^{2}}$ and $d_{3 z^{2}-r^{2}}$, which we designate $x$ and $z$ respectively. Hopping between them takes place through virtual jumps to the intermediate $\mathrm{O}$ ions. Fixing the orientations of the $e_{g}$ orbitals to the frame of reference of the lattice, we obtain for the $z$ - direction, $t_{z z}=t$ and $t_{x z}=t_{x x}=0$. For the directions in the $x-y$ plane $t_{z z}=\frac{1}{4} t, t_{x z}= \pm \frac{\sqrt{3}}{4} t$ and $t_{x x}=\frac{3}{4} t$, where the two signs in $t_{x z}$ correspond to the $x$ and $y$ directions, and $t$ is the effective $e_{g}-e_{g}$ hopping generated from the $(d p \sigma)$ matrix element between a $d$ orbital in a given $\mathrm{Mn}$ ion, and a $p$ orbital in a neighboring $\mathrm{O}$ ion [18,19]. At each site there is also a spin, from the three $t_{2 g}$ orbitals, which we treat as classical, and parametrize in terms of the Euler angles $\theta$ and $\phi$. We assume that the Hund's coupling between the $e_{g}$ electrons and this spin is much larger than other scales in the model. Then, the hopping elements depend on the orientation of the core spins, and the actual hopping is 20]:

$$
t_{\alpha, \beta}^{i, j}=t_{\alpha, \beta}\left(\cos \frac{\theta_{i}}{2} \cos \frac{\theta_{j}}{2}+\sin \frac{\theta_{i}}{2} \sin \frac{\theta_{j}}{2} e^{i\left(\phi_{i}-\phi_{j}\right)}\right)
$$

where the value of the hopping has been estimated $t \sim$ $0.1-0.3 \mathrm{eV}$.

We study a cubic lattice with periodic boundary conditions in the $x$ and $y$ directions, and open boundary conditions along the $z$ direction, where two (001) surfaces terminate the lattice. We use slabs with a thickness of 20 atoms. We have checked that, for this size, the bulk properties are recovered at the center of the slab. The environment of the outermost Mn ions is deficient in oxygen. The oxygen octahedra which surround the Mn ions are incomplete. The absence of the negatively charged $\mathrm{O}^{2-}$ ions leads to a downward shift of the $e_{g}$ levels with respect to the values in the bulk. This shift is larger for the $d_{3 z^{2}-r^{2}}$ orbital, which points towards the surface. The $d_{x^{2}-y^{2}}$ orbital is more localized around the ion, and is less sensitive to the change in the environment. In order to keep the number of free parameters in the model to a minimum, we leave the $d_{x^{2}-y^{2}}$ level unchanged with respect to the bulk, while the $d_{3 z^{2}-r^{2}}$ is shifted downwards by an amount $\Delta$. The value of $\Delta$ should be comparable, or larger, than the observed Jahn-Teller splitting 
in $\mathrm{LaMnO}_{3}$, which, in turn, is larger than the $e_{g}$ bandwidth. Reasonable values of $\Delta$ are $\sim 0.5-1.5 \mathrm{eV}$ [2]. The $e_{g}$ levels at all other layers remain unchanged, except for electrostatic effects (see below).

This shift of the surface $d_{3 z^{2}-r^{2}}$ orbitals leads to charge transfer between the bulk and the surface. We treat the induced electrostatic effects within the Hartree approximation. This gives rise to an additional shift in the electronic levels which is determined by solving selfconsistently the Schrödinger and the Poisson equations. This electrostatic shift is equal for the two $e_{g}$ orbitals at each layer. Screening from other levels is described in terms of a dielectric constant of value $\epsilon=5 \epsilon_{0}$. Selfconsistency is imposed on the $e_{g}$ levels in all layers in the system. We do not consider the possibility of orbital ordering induced by a Hubbard $U$ between electrons at the two $e_{g}$ orbitals [21]. We analyze underdoped materials, $x<0.5$, where no unusual magnetic textures are expected [22]. The Hartree approximation acts to suppress charge fluctuations, although it does not split the $e_{g}$ bands in the way a Hubbard term does. This effect should be less important at the surface, due to the crystal field splitting $\Delta$ introduced before.

Typical results, for $\Delta=10 t$, hole concentration of $x=0.3$ and a ferromagnetic configuration of all spins are shown in fig. [1]. Charge neutrality corresponds to a total occupancy of the $e_{g}$ orbitals of 0.7 .

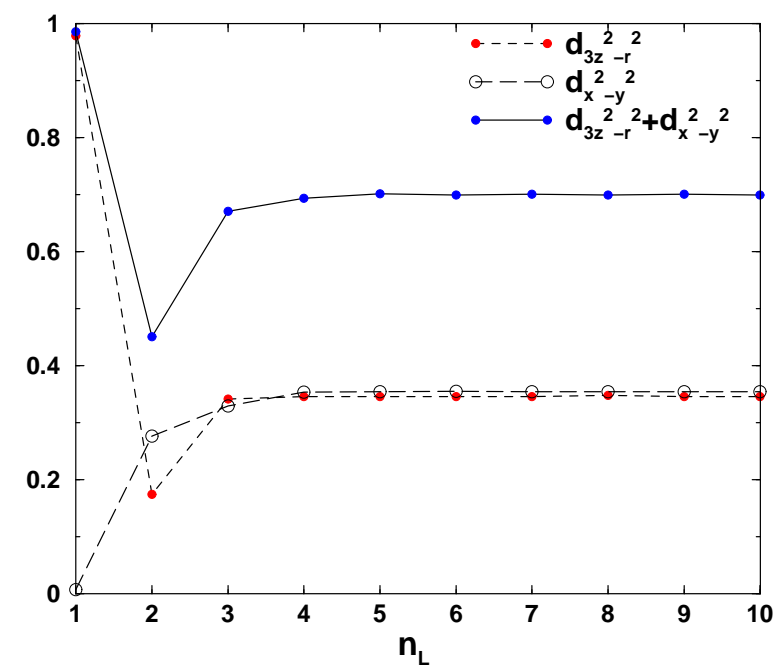

FIG. 1. Charge in the $e_{g}$ orbitals as a function of the distance from the surface for $\Delta=10 t$. On the surface, $n_{L}=1$, the $x$ orbitals are almost empty, due to the shift $\Delta$.

The large splitting between the $e_{g}$ orbitals at the surface leads to a significant charge transfer to the outermost layer. The charge distribution reaches the bulk values at the third layer, in agreement with the expected short screening length of the metal. The surface $d_{3 z^{2}-r^{2}}$ orbitals are almost full, while the $d_{x^{2}-y^{2}}$ are empty, so that the charge state of the ion is $\mathrm{Mn}^{3+}$. Hence, for the value of $\Delta$ used in fig. [1], the double exchange mechanism is almost completely suppressed at the surface. Higher val- ues of $\Delta / t$ do not alter these results. For intermediate values of $\Delta / t$, the surface electrostatic barrier depends significantly on the magnetic configuration. The surface $d_{x^{2}-y^{2}}$ orbitals are practically empty, and the $d_{3 z^{2}-r^{2}}$ is occuppied for $\Delta>3 t$.

The suppression of the double exchange ferromagnetic coupling at the surface leads to an enhancement of the superexchange interaction among the Mn ions. To investigate further this effect, we study magnetic configurations where the core spins of the surface $\mathrm{Mn}$ ions are allowed to rotate, as shown in fig. [2]. The canting angle $\theta$ is used as a variational parameter. $\theta=0$ implies a perfect ferromagnetic order at the surface. $\theta=\pi / 2$ gives rise to an antiferromagnetic alignment of the surface spins, at right angles with the bulk magnetization. To take into account the superexchange interaction, we introduce an antiferromagnetic coupling between the Mn core spins. Substracting a trivial constant, the energy per surface ion, due to this coupling, is:

$$
E_{A F}=J_{\perp} \cos (\theta)+2 J_{\|} \cos (2 \theta)
$$

where $\theta$ is the angle shown in fig. [2], $J_{\perp}$ is the antiferromagnetic coupling between a $\mathrm{Mn}$ spin at the surface and one in the next layer, and $J_{\|}$is the coupling between spins at the surface layer. From symmetry considerations, $J_{\|}=4 J_{\perp} 23$.

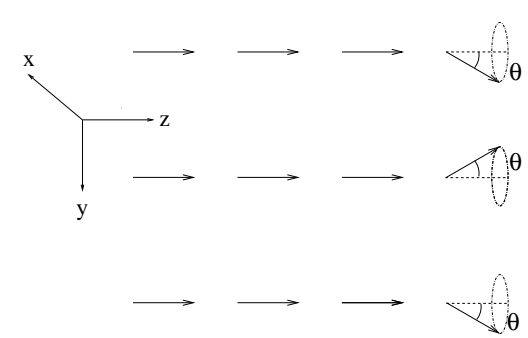

FIG. 2. Magnetic structure considered in the text. Only the spins at the surface layer are rotated, in the way shown.

The total energy of the system is the sum of the kinetic, Hartree and magnetic energies. By minimizing the total energy as a function of $\theta$ we obtain the canting angle as a function of the antiferromagnetic coupling $J_{\perp}$.

Fig. [3] shows the calculated phase diagram as function of the splitting at the surface between the $e_{g}$ levels, $\Delta$, and the direct antiferromagnetic coupling between the core spins $J_{\perp}$, for two different values of the hole concentration. 


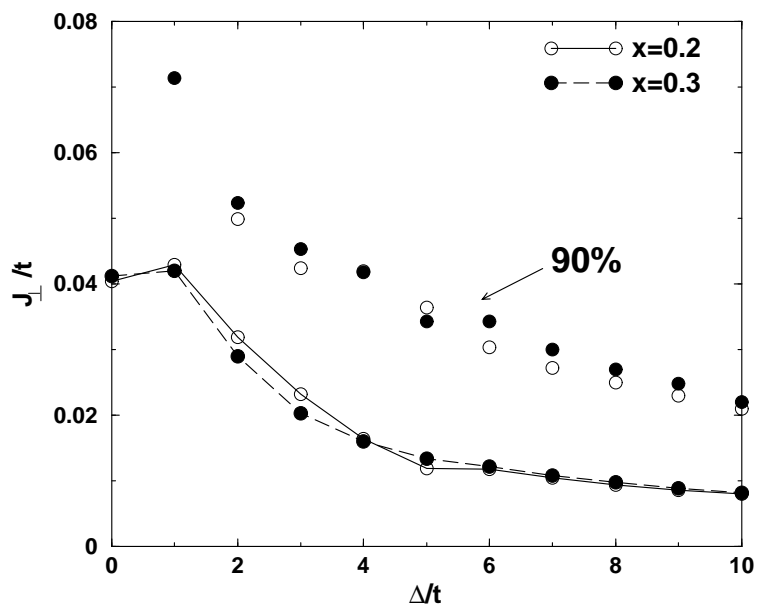

FIG. 3. Magnetic phase diagram of the surface of a doped manganite. The lines separate the fully ferromagnetic and the canted regions. The dots indicate that the order is $90 \%$ antiferromagnetic, namely, $\theta=81^{\circ}$. Full dots are results for hole concentration of $x=0.3$. Open dots are for $x=0.2$.

Realistic values of $J_{\perp}$ are of the order of $0.02 t$ [24] and from fig. [3], we see that, for this value, the value of $\theta$ is close to that of perfect surface antiferromagnetism, $\pi / 2$. Our approach tends to underestimate this tendency towards antiferromagnetism, as we do not allow to relax the spins in the layers deeper into the surface.

The changes in the magnetic surface structure also lead to modifications in the spin stiffness at the surface, which is weaker than in the ferromagnetic bulk. It is straightforward to show that weaker couplings lead to the formation of bands of surface magnons [25]. We find such bands both in the canted and in the ferromagnetic phases shown in fig. [3]. The bandwidth is narrower than the bulk magnon band. Thermal excitation of these modes leads to a decay of the surface magnetization as function of temperature which is faster than in the bulk. In order to estimate this effects, we have calculated, using Monte Carlo techniques [26], the surface magnetization of a cluster of classical spins. In this model, the bulk double exchange mechanism is described by an effective ferromagnetic Heisenberg coupling $J$, and the surface spins interact with an antiferromagnetic coupling $J_{1}$. The outermost spins interact with the spins in the second layers via a ferromagnetic coupling $J_{2}$. From the results reported previously, we estimate that $J_{1}$ is very small, $\sim-J / 100$ and $J_{2}$ takes a value between $J / 2$ and $J / 6$. In fig. [4] we plot the surface magnetization as a function of the temperature for different values of $J_{2}$. The results obtained do not depend strongly on the value of $J_{2}$ and they are in reasonable agreement with the experimental results reported in 27.

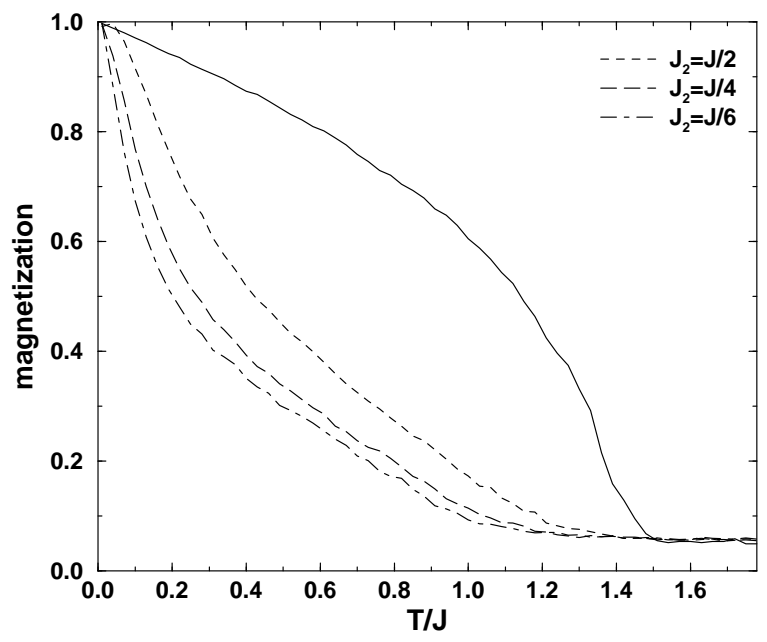

FIG. 4. Magnetization of the last layer as function of temperature for a cluster with 20 layers and open boundary conditions. For comparison, the results for a cluster of the same size and periodic boundary conditions (no surfaces) are also shown.

The combination of energy shifts and changes in the magnetic couplings reduces the transmission of the surface, even in the absence of other, extrinsic, barriers. In order to estimate this effect, we have calculated the resistance between two perfect double exchange ferromagnetic metals separated by a layer with the amount of charge and the magnetic structure obtained in the previous calculations. Since only the $d_{3 z^{2}-r^{2}}$ orbitals contribute to the transport through the interface, we have simplified the model, and only a single orbital per site is kept. The calculations were done using the method described in [28,29]. Two effects contribute to the resistivity, the shift in energy of the interface orbital and the difference in spin orientation between the atoms at the bulk and at the interface. The conductance is sharply reduced when the surface layer is antiferromagnetic, as the double exchange mechanism is suppressed. We find that for hole concentrations of $x=0.3$ the presence of the interface increases the resistance of the system in a factor bigger than 10 .

A magnetic field will reduce the antiferromagnetism at the surface, and, at high fields, the surface spins are aligned parallel to the bulk. We estimate this effect by adding a magnetic field to the model and finding the magnetic structure which minimizes the energy. The resulting conductance, for $\Delta=3 t$ and $J_{\perp}=0.025 t$, is plotted in fig. [5]. A very high field $\sim 60 \mathrm{~T}$ is required to saturate the magnetoresistance. Note that the low field ( $i$ 1T ) magnetoresistance is probably due to the alignment of the polarization of the bulk electrodes, which can be understood within conventional models [30,31]. Our results for the high field dependence are consistent with the available experimental data data [3 10]. 


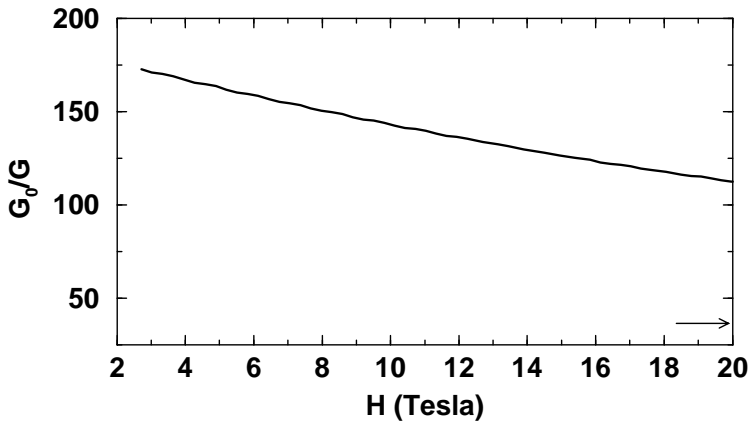

FIG. 5. Inverse conductance, normalized to the conductance of a perfect ferromagnetic system $(\theta=0$ and $\Delta=0)$, of a magnetic layer, as function of applied field. The arrow indicates the saturation limit at high fields. The size of the system is $20 \times 20 \times 20$. The parameters used are described in the text.

The magnetic excitations of the surface layer also modify the magnetoresistance at finite temperatures [32 34. Spin flip scattering due to thermally excited magnons leads to a suppression of the magnetoresistance at temperatures below the bulk Curie temperature. The dependence of the magnetoresistance on temperature should be similar to that of the surface magnetization, shown in fig. 近.

In conclusion, we have shown that the lack of cubic symmetry at surfaces, combined with the double exchange mechanism, leads to significant changes in the magnetic and transport properties of doped manganites. Charge is transferred from the bulk to the surface layers, and an antiferromagnetic ordering of the surface spins is favored. This structure is consistent with the observed reduction of the magnetoresistance in junctions and ceramic materials at temperatures below the bulk Curie temperature, with the high field dependence of the magnetoresistance and with the experimental results on surface magnetization.

We acknowledge fruitful discussions with A. de Andrés, M. Coey, M. Hernández, J. L. Martínez, J. Fontcuberta, $\mathrm{X}$. Obradors and J. A. Vergés. This research is supported through grants PB96/0875 and PB96/0085. LB amd MJC also acknowledge finantial support from the Fundación Ramón Areces.

[1] E. D. Wollan et al, Phys. Rev. 100, 545 (1955).

[2] J. M. D. Coey et al, Adv. in Phys., in press.

[3] H. Y. Hwang et al, Phys. Rev. Lett. 77, 2041 (1996).

[4] Y. Lu et al, Phys. Rev. B 54, R8357 (1996).

[5] A. Gupta et al, Phys. Rev. B 54, R15629 (1996).

[6] N. D. Mathur et al/, Nature 387, 266 (1997).

[7] M. Viret et al, Europhys. Lett. 39, 545 (1997).

[8] J. Z. Sun et al, Appl. Phys. Lett. 73, 1008 (1998).

[9] K. Steenbeck et al, Appl. Phys. Lett. 73, 2506 (1998).
[10] J. Z. Sun et al, Thickness dependent magnetotransport in ultra-thin manganite films, preprint (cond-mat/9809414).

[11] R. D. Sánchez et al, Appl. Phys. Lett. 68, 134 (1996).

[12] R. Mahesh et al, Appl. Phys. Lett. 68, 2291 (1996).

13] LL. Balcells et al, J. Phys. (cond. mat.) 10, 1883 (1998).

[14] J. Fontcuberta et al, J. Appl. Phys. 83, 7058 (1998).

[15] Ll. Balcells et al, Phys. Rev. B, in press.

[16] H. Y. Hwang and S.-W. Cheong, Nature 389, 942 (1997).

[17] M. Rajeswari et al, Appl. Phys. Lett. 73, 2672 (1998).

[18] D. A. Papaconstantopoulos and W. E. Pickett, Phys. Rev. B 57, 12751 (1998).

[19] D.Feinberg et al, Phys.Rev.B 57 R5583 (1998).

[20] E.Muller-Hortmann et al, Phys.Rev.B 54 R6819 (1996).

[21] D. I. Khomskii and G. Sawatzky, Solid State Commun. 102, 87 (1997).

[22] J.

van den Brink and D. Khomskii, Double-exchange via degenerate orbitals, preprint (cond-mat/9810394).

[23] A. J. Millis, Phys. Rev. B 55, 6405 (1997).

[24] T.G.Perring et al, Phys.Rev.Lett. 78,3197 (1997).

[25] D. C. Mattis, The Theory of Magnetism I : Statics and Dynamics, Springer, New York (1988).

[26] M.J.Calderón and L.Brey, Phys.Rev.B 58, 3284 (1998).

[27] J.-H. Park et al, Phys. Rev. Lett. 81, 1953 (1998).

[28] M. J. Calderón, J. A. Vergés and L. Brey, Conductance as a Function of the Temperature in the Double Exchange Model, preprint ( cond-mat/9806157).

[29] J. A. Vergés, Phys. Rev. B 57, 870 (1998).

[30] M. Julliere, Phys. Lett. 54A, 225 (1975).

[31] J. C. Slonczewski, Phys. Rev. B 39, 6995 (1989).

[32] A. M. Bratkovsky, Phys. Rev. B 56, 2344 (1997).

[33] F. Guinea, Phys. Rev. B 58, 9212 (1998).

[34] J. S. Moodera et al, Phys. Rev. Lett. 80, 2941 (1998). 